\title{
Proceeding
}

10th INSHS International Christmas Sport Scientific Conference, 4-5 December 2015. International Network of Sport and Health

Science. Szombathely, Hungary

\section{Postural and balance evaluation in 18-30 years old albaniana $\beta$-thalassemia patients}

\author{
GENTI PANO 1 , ROBERT ÇINA ${ }^{2}$, GENTI MURATAJ ${ }^{2}$, ALJONA KRISTULI ${ }^{2}$, DEA RUSI ${ }^{2}$ \\ ${ }^{1}$ Department of PA Health and Recreation, Sports University of Tirana, Tirana Albania \\ ${ }^{2}$ Department of Sport Medicine, Sports University of Tirana, Tirana Albania
}

\begin{abstract}
According to the World Health Organization (WHO), there are at least 70 million thalassemia carriers in the world and in each year 42,000 homozygote children are born. The bone abnormalities such as rickets, spinal deformities, severe osteoporosis, and pathological fractures are important morbidity causes (Rodda CP et al., 1995). Patients with well treated transfusion-dependent thalassemia are generally shorter, have reduced truncal height (Fung EB et al., 2010), and healthy body weight (Vichinsky EP., 1998) compared to the general population. The main objective of this study was to evaluate postural and balance problems in beta thalassemia patients. 28 subjects (7 males 21 females) 18-30 years old from 3 different epidemiologic cities in Albania have participated in this study. Postural screening, which included digital photography in 4 plans (anterior view, right lateral view, posterior view, left lateral view) and postural evaluation with Posture Screen Mobile program were done. Subjects also performed balance tests on the Leonardo Mechanography Platform in 4 different position; BT (Balance Test) 1. (Rom EO); 1a (Rom EC); 2. (SemTanEO); 2a (SemTanEC); 3. (TanEO); 3a (TanEC). Pearson Correlation was used to asses' associations between head lateral translations and angulations. Based on the results we can say that in beta thalassemia patients the postural problems are a major health related problem. More studies focused on this category are necessary in order to evaluate the most effective PA program. As a very specific group population it is recommended that the postural evaluation need to be part of their health routine check-up in order to prevent degenerative major postural deviations. Key words: POSTURE, BALANCE, POSTURAL ADJUSTMENTS.
\end{abstract}

Corresponding author. Sports University of Tirana, Sport Sciences Research Institute, Department of PA Health and Recreation, Tirana Albania.

E-mail: genti.pano@outlook.com

10th INSHS International Christmas Sport Scientific Conference, 4-5 December 2015. International Network of Sport and

Health Science. Szombathely, Hungary.

JOURNAL OF HUMAN SPORT \& EXERCISE ISSN 1988-5202

(c) Faculty of Education. University of Alicante

doi:10.14198/jhse.2016.11.Proc1.17

VOLUME 11 | Proc1 | 2016 | S251 


\section{INTRODUCTION}

According to the World Health Organization (WHO), there are at least 70 million thalassemia carriers in the world and in each year 42,000 homozygote children are born. The bone abnormalities such as rickets, spinal deformities, severe osteoporosis, and pathological fractures are important morbidity causes (Rodda CP et al., 1995). Patients with well treated transfusion-dependent thalassemia are generally shorter, have reduced truncal height (Fung EB et al., 2010), and healthy body weight (Vichinsky EP., 1998) compared to the general population. Muscular stability and mobility, skeletal structure, and muscular balance all influence postural alignment (Kendall, F.P et al., 1983; Fortin, C et al., 2011). Depending on the severity of the postural deviations, poor posture may result in pain and can affect physical function and the ability to perform activities of daily living (Katzman, W.B., et al., 2007). Regarding postural measurement methods, there are several tools available for clinical use. These include simple photographic techniques and plumb-line measures (Zonnenberg AJJ et al., 1996; Raine S, Twomey LT., 1997; Vernon H., 1983; Bullock-Saxton J et al., 1993), simple goniometers, inclinometers and linear devices (Braun BL, Amundson LR., 1989; Grimmer K., 1997; Nilsson BM, Soderlund A., 2005), placing known sized blocks between postural regions (Grimmer K., 1997), various computer assisted methods including electro-goniometers (Christensen HW, Nilsson N., 1999), electromagnetic movement systems (Swinkels A, Dolan P ., 2000; Swinkels A, Dolan P 2., 1998), computer assisted digitization systems (Dunk NM et al., 2004; Dunk NM et al., 2005; Beaudoin L et al., 1999), and 3D ultrasound-based motion analysis device for the cervical spine (Strimpakos $\mathrm{N}$ et al., 2005). PostureScreenMobile is a relatively new program that can be found in App Store or Apple Store, that makes the assessment of posture in a variety of settings more efficacious. PostureScreenMobile is designed for chiropractors, physical and manual therapists and other fitness professionals that screen clients for postural deviations.

\section{Objectives}

The main objective of this study was to evaluate postural and balance in Albanian $\beta$-thalassemia patients.

\section{MATERIAL AND METHODS}

A total of $28 \beta$-thalassemia subjects (7 males 21 females) 18-30 years old from 3 different epidemiologic cities in Albania have participated in this study. Postural screening, which included digital photography in 4 plans (anterior view, right lateral view, posterior view, left lateral view) and postural evaluation with Posture Screen Mobile program were done. was also performed on Leonardo Mechanography Platform a set of 3 balance tests BT (Balance Test). The subject is trying to stand in a quiet normal standing poition for a certain amount of time (exmple $10 \mathrm{sec}$ ). For the test to be more difficult are used and we have used 2 other different foot positions, conducted with open eyes and closed eyes. Statistical analyses were performed using IBM SPSS Statistics version 20.

\section{BT (Balance Test $10 \mathrm{sec}$ ) protocol}

1. Romberg standing position with Eyes Open; 1a Romberg standing position Eyes Closed (Rom EO; RomEC).

2. Semi Tangent position with Eyes Open; 2a. Semi Tangent position with Eyes closed (SemTanEO); (SemTanEC).

3. Tangent position with Eyes Open; 3a. Tangent position with Eyes Closed (TanEO); (TanEC). 


\section{RESULTS AND DISCUSSION}

\section{Posture results}

Table 1. Posture Displacement. Anterior View and Right Lateral View

\begin{tabular}{lcccc} 
N=21 & $\begin{array}{c}\text { Anterior } \\
\text { Translations }\end{array}$ & $\begin{array}{c}\text { Anterior } \\
\text { Angulations }\end{array}$ & $\begin{array}{c}\text { Lateral } \\
\text { Translations }\end{array}$ & $\begin{array}{c}\text { Lateral } \\
\text { Angulations }\end{array}$ \\
\hline Mean & 1.45 & 5.95 & 4.57 & 21.44 \\
Std. deviation & 0.58 & 3.52 & 2.46 & 8.77 \\
Skew & 0.77 & 2.02 & 2.26 & 0.58 \\
Kurtosis & 0.41 & 6.54 & 7.39 & -0.43 \\
\hline
\end{tabular}

Table 2. Posture Displacement. Anterior View and Left Lateral View

\begin{tabular}{lcccc} 
N=21 & $\begin{array}{c}\text { Posterior } \\
\text { Translations }\end{array}$ & $\begin{array}{c}\text { Posterior } \\
\text { Angulations }\end{array}$ & $\begin{array}{c}\text { Lateral } \\
\text { Translations }\end{array}$ & $\begin{array}{c}\text { Lateral } \\
\text { Angulations }\end{array}$ \\
\hline Mean & 3.11 & 20.42 & 4.46 & 22.93 \\
Std. deviation & 1.28 & 12.45 & 2.27 & 8.66 \\
Skew & 1.28 & 2.09 & 1.35 & 0.42 \\
Kurtosis & 3.24 & 6.68 & 2.49 & 0.04 \\
\hline
\end{tabular}

Table 3. Head region results

\begin{tabular}{lcc}
$\mathbf{N}=\mathbf{2 1}$ & $\begin{array}{c}\text { Lateral } \\
\text { Translations }\end{array}$ & $\begin{array}{c}\text { Lateral } \\
\text { Angulations }\end{array}$ \\
\hline Mean & 1.28 & 11.78 \\
Std. deviation & 0.66 & 7.17 \\
Skew & 0.61 & 1.15 \\
Kurtosis & -0.63 & 1.57 \\
\hline
\end{tabular}

Table 4. Shoulder region results

\begin{tabular}{lcc} 
N=21 & $\begin{array}{c}\text { Lateral } \\
\text { Translations }\end{array}$ & $\begin{array}{c}\text { Lateral } \\
\text { Angulations }\end{array}$ \\
\hline Mean & 1.23 & 2.16 \\
Std. deviation & 0.87 & 1.75 \\
Skew & 1.31 & 0.97 \\
Kurtosis & 1.67 & -0.02 \\
\hline
\end{tabular}


Table 5. Hip Pelvis region results

\begin{tabular}{lcc} 
N=21 & $\begin{array}{c}\text { Lateral } \\
\text { Translations }\end{array}$ & $\begin{array}{c}\text { Lateral } \\
\text { Angulations }\end{array}$ \\
\hline Mean & 1.05 & 3.63 \\
Std. deviation & 0.77 & 2.79 \\
Skew & 1.63 & 1.59 \\
Kurtosis & 2.38 & 3.39 \\
\hline
\end{tabular}

Table 6. Knee region results

\begin{tabular}{lcc}
$\mathbf{N}=\mathbf{2 1}$ & $\begin{array}{c}\text { Lateral } \\
\text { Translations }\end{array}$ & $\begin{array}{c}\text { Lateral } \\
\text { Angulations }\end{array}$ \\
\hline Mean & 0.98 & 3.69 \\
Std. deviation & 0.62 & 1.69 \\
Skew & 2.50 & -0.14 \\
Kurtosis & 9.11 & -1.07 \\
\hline
\end{tabular}

Head lateral translations and angulations were tested against normal distribution using Shapiro Wilk test of normality. Shapiro Wilk test and box plot results demonstrated that Head lateral translations and angulations scores distribution met the normality requirements. Normality box plots are presented below:

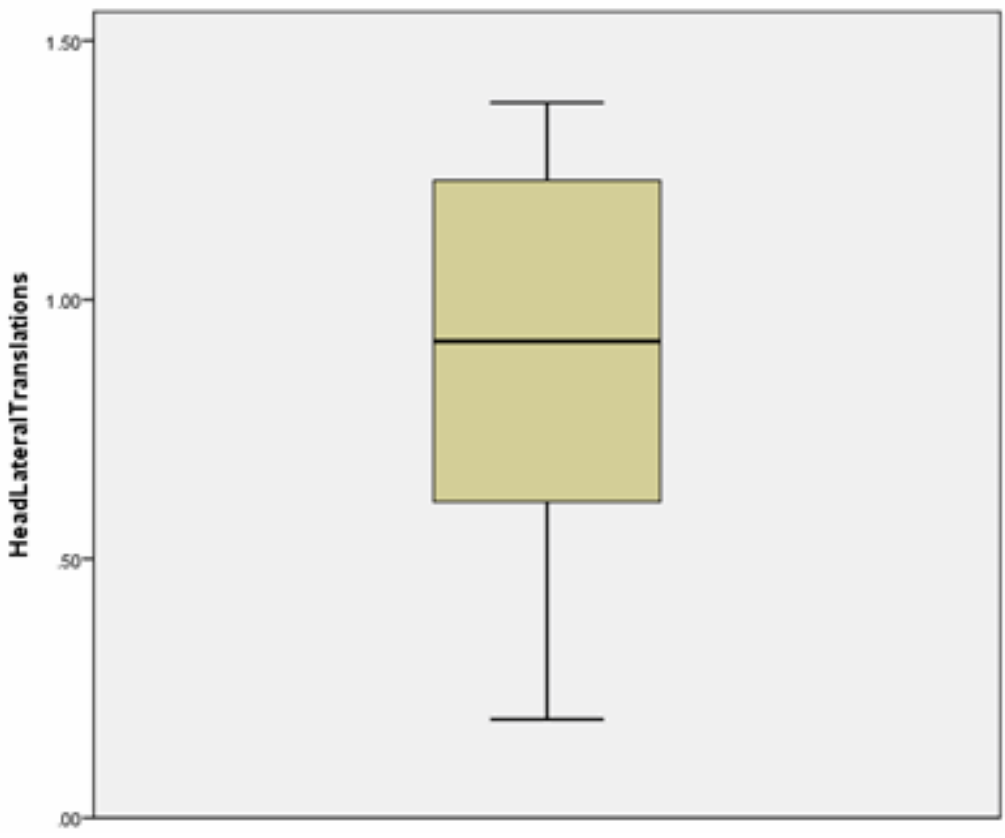

Figure 1. Head Lateral Translations results 


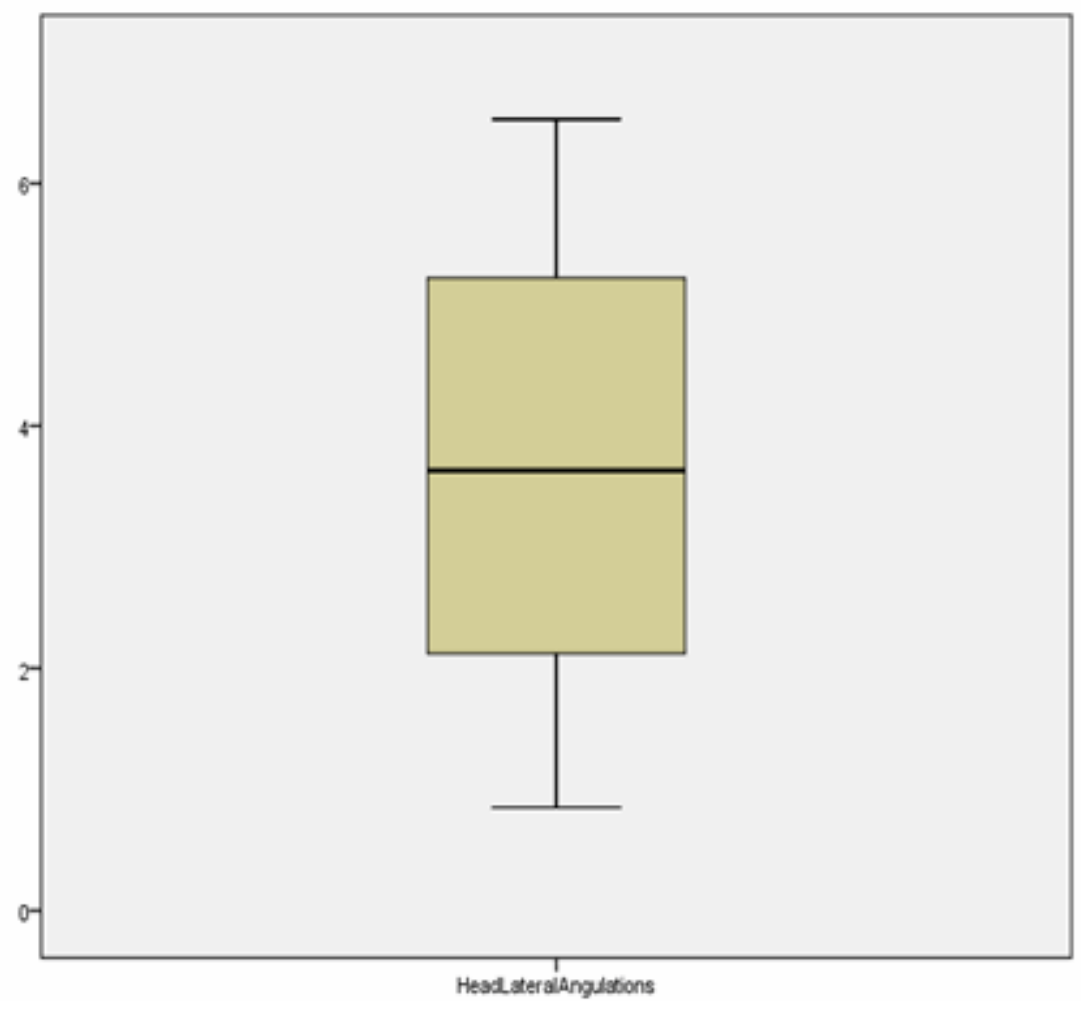

Figure 2. Head Lateral Angulations results

Pearson Correlation was used to asses' associations between head lateral translations and angulations. Pearson 's correlation $(21)=0.804, p<0.0005$ ) revealed a strong positive and significant correlation between head lateral translations and angulations.

Table 7. Correlations

\begin{tabular}{|c|c|c|c|}
\hline & & $\begin{array}{l}\text { Head Lateral } \\
\text { Translations }^{\circ}\end{array}$ & $\begin{array}{l}\text { Head Lateral } \\
\text { Angulations }\end{array}$ \\
\hline \multirow{4}{*}{ Head Lateral Translations } & Pearson Correlation & 1 & $.804^{88}$ \\
\hline & Sig. (2-tailed) & & .000 \\
\hline & $\mathrm{N}$ & 21 & 21 \\
\hline & Pearson Correlation & $.804^{88}$ & 1 \\
\hline \multirow[t]{2}{*}{ Head Lateral Angulations } & Sig. (2-tailed) & .000 & \\
\hline & $\mathrm{N}$ & 21 & 21 \\
\hline **. Correlation is significar & 0.01 level (2-tailed). & & \\
\hline
\end{tabular}




\section{Balance results}

Table 8. Balance Test (BT) RomEO). Analysis Results, Balance Data

\begin{tabular}{|c|c|c|c|c|c|c|c|c|c|c|}
\hline & Age & $\begin{array}{l}\text { Body } \\
\text { Mass }\end{array}$ & $\begin{array}{l}\text { Std. } \\
\text { Elipse } \\
\text { Area } \\
\mathrm{cm}^{2}\end{array}$ & $\begin{array}{l}\text { Std. } \\
\text { Elipse } \\
\text { Angle }\end{array}$ & $\begin{array}{l}\text { num. } \\
\text { Eccentricity }\end{array}$ & $\begin{array}{l}\text { dominat } \\
\text { Freq. } \\
\mathrm{Hz}\end{array}$ & $\begin{array}{l}\text { rel. Path } \\
\text { length } \\
\mathrm{mm} / \mathrm{s}\end{array}$ & $\begin{array}{l}\text { abs. } \\
\text { Path } \\
\text { length } \\
\text { mm }\end{array}$ & $\begin{array}{l}\text { EQ } \\
\text { (AP) }\end{array}$ & $\begin{array}{l}\text { Total } \\
\text { Duration } \\
\mathrm{s}\end{array}$ \\
\hline Mean & 22.3 & 53.22 & 0.77 & 16.74 & 0.85 & 0.47 & 15.05 & 75.3 & 94.89 & 5 \\
\hline
\end{tabular}

Table 9. Balance Test (BT) RomEC). Analysis Results, Balance Data

\begin{tabular}{|c|c|c|c|c|c|c|c|c|c|c|}
\hline & Age & $\begin{array}{l}\text { Body } \\
\text { Mass }\end{array}$ & $\begin{array}{l}\text { Std. } \\
\text { Elipse } \\
\text { Area } \\
\mathrm{cm}^{2}\end{array}$ & $\begin{array}{l}\text { Std. } \\
\text { Elipse } \\
\text { Angle. }\end{array}$ & $\begin{array}{l}\text { num. } \\
\text { Eccentricity }\end{array}$ & $\begin{array}{l}\text { dominat } \\
\text { Freq. } \\
\mathrm{Hz}\end{array}$ & $\begin{array}{l}\text { rel. Path } \\
\text { length } \\
\mathrm{mm} / \mathrm{s}\end{array}$ & $\begin{array}{l}\text { abs. } \\
\text { Path } \\
\text { length } \\
\text { mm }\end{array}$ & $\begin{array}{l}E Q \\
\text { (AP) }\end{array}$ & $\begin{array}{l}\text { Total } \\
\text { Duration } \\
\mathrm{s}\end{array}$ \\
\hline Mean & 22.3 & 53.22 & 1.87 & 3.04 & 0.80 & 0.58 & 20.78 & 103.93 & 91.88 & 5 \\
\hline
\end{tabular}

Table 10. Balance Test (BT) TanEO). Analysis Results, Balance Data

\begin{tabular}{|c|c|c|c|c|c|c|c|c|c|c|}
\hline & Age & $\begin{array}{l}\text { Body } \\
\text { Mass }\end{array}$ & $\begin{array}{l}\text { Std. } \\
\text { Elipse } \\
\text { Area } \\
\mathrm{cm}^{2} \\
\end{array}$ & $\begin{array}{l}\text { Std. } \\
\text { Elipse } \\
\text { Angle }\end{array}$ & $\begin{array}{l}\text { num. } \\
\text { Eccentricity }\end{array}$ & $\begin{array}{l}\text { dominat } \\
\text { Freq. } \\
\mathrm{Hz}\end{array}$ & $\begin{array}{l}\text { rel. Path } \\
\text { length } \\
\mathrm{mm} / \mathrm{s}\end{array}$ & $\begin{array}{l}\text { abs. } \\
\text { Path } \\
\text { length } \\
\text { mm }\end{array}$ & $\begin{array}{l}E Q \\
(A P)\end{array}$ & $\begin{array}{l}\text { Total } \\
\text { Duration } \\
\mathrm{s}\end{array}$ \\
\hline Mean & 22.3 & 53.22 & 29.66 & 0.29 & 0.88 & 0.47 & 76.07 & 760.84 & 70.33 & 10 \\
\hline
\end{tabular}

Table 11. Balance Test (BT) TanEC). Analysis Results, Balance Data

\begin{tabular}{|c|c|c|c|c|c|c|c|c|c|c|}
\hline & Age & $\begin{array}{l}\text { Body } \\
\text { Mass }\end{array}$ & $\begin{array}{l}\text { Std. } \\
\text { Elipse } \\
\text { Area } \\
\mathrm{cm}^{2}\end{array}$ & $\begin{array}{l}\text { Std. } \\
\text { Elipse } \\
\text { Angle }\end{array}$ & $\begin{array}{l}\text { num. } \\
\text { Eccentricity }\end{array}$ & $\begin{array}{l}\text { dominat } \\
\text { Freq. } \\
\mathrm{Hz}\end{array}$ & $\begin{array}{l}\text { rel. Path } \\
\text { length } \\
\mathrm{mm} / \mathrm{s}\end{array}$ & $\begin{array}{l}\text { abs. } \\
\text { Path } \\
\text { length } \\
\mathrm{mm}\end{array}$ & $\begin{array}{l}\mathrm{EQ} \\
(\mathrm{AP})\end{array}$ & $\begin{array}{l}\text { Total } \\
\text { Duration } \\
\mathrm{s}\end{array}$ \\
\hline Mean & 22.3 & 53.22 & 3.073 & -3.7 & 0.88 & 0.69 & 42.64 & 213.21 & 86.13 & 5 \\
\hline
\end{tabular}

Results show that the major part of the subjects especially the males, had serious postural problems due to disease side effects such as high levels of calcium due to transfusion, inactivity due to lack of appropriate muscle mass and all the other health relates disease conditions. Also Balance test results also show that the majority of the subjects have balance problems especially in the eye closed tests which according to the results is related with poor posture.

\section{CONCLUSIONS}

Based on the results we can say that in beta thalassemia patients the postural problems are a major health related problem. As a very specific group population it is recommended that the postural evaluation need to be part of their health routine check-up in order to prevent degenerative major postural deviations. Also it is necessary to conduct further research with larger subjects and different age-groups in order to have more reliable results regarding posture and balance in this category for better evaluate and describe more effective physical activity exercise programs to prevent or correct possible postural and balance problems. 


\section{REFERENCES}

1. Beaudoin, L., Zabjek, K.F., Leroux, M.A., Coillard, C., Rivard, C.H. (1999). Acute systematic and variable postural adaptations induced by an orthopaedic shoe lift in control subjects. Eur. Spine J., $8,40-45$.

2. Braun, B.L., Amundson, L.R. (1989). Quantitative assessment of head and shoulder posture. Arch. Phys. Med. Rehabil., 70, 322-9.

3. Bullock-Saxton, J. (1993). Postural alignment in standing: a repeatable study. Austr. Physiother., 39, 25-29.

4. Christensen, H.W., Nilsson, N. (1990). The ability to reproduce the neutral zero position of the head. J. Manipulative Physiol. Ther., 9(22), 26-28.

5. Dunk, N.M., Chung, Y.Y., Compton, Callaghan, J.P. (2004). The reliability of quantifying upright standing postures as a baseline diagnostic clinical tool. J. Manipulative Physiol. Ther., 27, 91-96.

6. Dunk, N.M., Lalonde, J., Callaghan, J.P. (2005). Implications for the use of postural analysis as a clinical diagnostic tool: reliability of quantifying upright standing spinal postures from photographic images. J. Manipulative Physiol. Ther., 28, 386-392.

7. Fortin, C., et al. (2011). Clinical methods for quantifying body segment posture: A literature review. Disabil. Rehabil., 33(5), 367-83.

8. Fung, E.B., Xu, Y., Kwiatkowski, J.L., Vogiatzi, M.G., Neufeld, E., Olivieri, N., Vichinsky, E.P., Giardina, P.J. (2010). Relationship between chronic transfusion therapy and body composition in subjects with thalassemia. J. Pediatr., 157, 641-647

9. Grimmer, K. (1997). An investigation of poor cervical resting posture. Australian Physiotherapy, 43, 7-16.

10. $\mathrm{https}: / /$ itunes.apple.com/en/app/posturescreen-posture-body/id405109185?mt=8.

11. Katzman, W.B., et al. (2007). Changes in flexed posture, musculoskeletal impairments, and physical performance after group exercise in community-dwelling older women. Arch. Phys. Med. Rehabil., 88(2), 192-9.

12. Kendall, F.P., E.K. McCreary. (1983). Muscles, Testing and Function, 3rd ed., Baltimore: Lippincott, Willams \& Wilkins.

13. Nilsson, B.M., Soderlund, A. (2005). Head posture in patients with whiplash-associated disorders and the measurement method's reliability - A comparison to healthy subjects. Advances in Physiotherapy, 7, 13-19.

14. Raine, S., Twomey, L.T. (1997). Head and shoulder posture variations in 160 asymptomatic women and men. Arch. Phys. Med. Rehabil., 78, 1215-1221.

15. Rodda, C.P., Reid, E.D., Johnson, S., Doery, J., Matthews, R., Bowden, D.K. (1995). Short stature in homozygous $\beta$-thalassaemia is due to disproportionate truncal shortening. Clin. Endocrinol., 42, $587-592$

16. Strimpakos, N., Sakellari, V., Gioftsos, G., Papathanasiou, M., Brountzos, E., Kelekis, D., Kapreli, E., Oldham, J. (2005). Cervical spine ROM measurements: optimizing the testing protocol by using a 3D ultrasound- based motion analysis system. Cephalgia, 25, 1133-45.

17. Swinkels, A., Dolan, P. (1998). Regional assessment of joint position sense in the spine. Spine, 23, 590-597.

18. Swinkels, A., Dolan, P. (2000). Spinal position sense is independent of the magnitude of movement. Spine, 25, 98-105.

19. Vernon, H. (1983). An assessment of the intra- and inter-reliability of the posturometer. $J$. Manipulative and Physiol. Ther., 6, 57-60. 
20. Vichinsky, E.P.(1998). The morbidity of bone disease in thalassemia. Ann. NY. Acad. Sci., 8, 850344.

21. Zonnenberg. A.J.J., Maanen, V., Elvers, J.W.H., Oostendorp, R.A.B. (1996). Intra/interrater reliability of measurements on body posture photographs. J. Craniomandibular Practice, 14, 326-331. 